\title{
PAPR Reduction in STBC MIMO OFDM System using AMS and PTS Schemes
}

\author{
Arjun Chopra \\ School of Electronics and Communication \\ Engineering \\ Lovely Professional University, \\ Phagwara, Punjab, India
}

\author{
Komal Arora \\ Department of Electronics and Communication \\ Engineering \\ Lovely Professional University, \\ Phagwara, Punjab, India
}

\begin{abstract}
Increased data rates and reliability are the two key factors required to support emerging multimedia applications and new communications technologies. The two techniques used in high data rate transmission are orthogonal frequency division multiplexing (OFDM) and multiple-input multipleoutput (MIMO) scheme. The OFDM is used to mitigate the problem of inter symbol interference (ISI) and provides good protection against co-channel interference and noise. MIMO system helps to reduce fading and can be used for decreasing bit error rate that is spatial diversity or to increase the data rate that is spatial multiplexing. The combination of MIMO and OFDM is MIMO OFDM system. MIMO-OFDM system converts frequency selective MIMO channel into multiple parallel flat fading channels. One of the major drawbacks of in MIMO-OFDM systems is that the transmitted signal exhibits a high PAPR when the input sequences are correlated. In this paper, AMS and PTS schemes have been used to reduce peak to average power ratio (PAPR) in multiple input multiple output orthogonal frequency division multiplexing (MIMO OFDM) system with Alamouti's space time block coding (STBC). The AMS scheme reduces the computational complexity and when AMS scheme is used with quadrature amplitude modulation (QAM). Simulation and results show that the AMS scheme reduces PAPR more efficiently than the PTS scheme.
\end{abstract}

\section{Keywords}

Alternate Multisequence (AMS), Multiple Input Multiple output (MIMO), Orthogonal Frequency Division Multiplexing (OFDM), Space Time Block Codes (STBC), Partial Transmit Sequence (PTS), Quadrature Amplitude Modulation (QAM)

\section{INTRODUCTION}

With the advent of next generation (4G) broadband wireless communications, the combination of multiple-input multipleoutput (MIMO) wireless technology with orthogonal frequency division multiplexing (OFDM) has been recognized as one of the most promising techniques to support high data rate and high performance [1]. Multiple-input multiple-output (MIMO) wireless technology in combination with orthogonal frequency division multiplexing (OFDM) is an attractive airinterface solution for next-generation wireless local area networks (WLANs), wireless metropolitan area networks (WMANs), and fourth-generation mobile cellular wireless systems [2]. Multiple-input multiple-output (MIMO) technology meet the demands of next generation by offering increased spectral efficiency through spatial multiplexing gain, and improved link reliability due to antenna diversity gain [3]. OFDM has the potential to play an important role in the design of the next generation broadband wireless communication systems. Orthogonal frequency division multiplexing (OFDM) is a multi-carrier modulation technique that transmits the signals through multiple carriers and these multi-carriers or subcarriers have different frequencies. The major advantage of OFDM is its ability to cope with severe channel conditions, for example, multipath fading and narrowband interference, without complicated equalization filters [1]. One popular combination of MIMO and OFDM is the STBC-OFDM which was first proposed in [4, 5]. Alamouti-based space-time coding technique is one of the most effective transmitter diversity methods and when combined with OFDM, it enhances the system performance [6]. Space-time block coding (STBC) has come out as one of the major techniques to tap the MIMO benefit. STBC consists of data coded through space and time to improve the reliability of the transmission. An OFDM signal has high PAPR from the summation of the subcarriers at the transmitter and some combination of the subcarriers creates large peaks. The envelope of the transmitted signal is not constant showing peaks whose power exceeds the mean power. Therefore, to prevent the OFDM signal from distortion, the transmit amplifier must work in linear region. Therefore, for OFDM systems large range power amplifiers are required. But these amplifiers increase the cost of the OFDM systems. Consequently, reducing the PAPR is essential to reduce the cost of OFDM systems. Recently, various algorithms of the PAPR reduction have been proposed for MIMO OFDM systems in the literature, including clipping [7], selected mapping (SLM) [8], [9], partial transmit sequences (PTS) [10], [11]. However, when these methods are directly employed to reduce the PAPR in MIMO-OFDM systems, it results in an increase of the complexity and redundancy with the increasing number of antennas.

In this paper, an alternative multisequence (AMS) [12] and partial transmit sequence (PTS) scheme is used to reduce the PAPR of MIMO-OFDM signals. Alamouti space-time block coding (STBC) is employed in MIMO-OFDM systems in this paper with 4-QAM and 16-QAM.

\section{SYSTEM MODEL}

In this MIMO OFDM system, STBC codes are used as a channel coding technique to do error correction and detection and AMS/PTS scheme is employed to reduce PAPR. The input bits are given to modulator where modulation of input bits takes place using M-QAM complex constellation.

The modulated signal is given by

$$
\begin{aligned}
S_{m}(t)=A_{m c} g(t) \operatorname{Cos}\left(2 \pi f_{c} t\right) & \\
& -A_{m s} g(t) \operatorname{Sin}\left(2 \pi f_{c} t\right)
\end{aligned}
$$


$A_{m c}$ and $A_{m s}$ are information bearing signal amplitudes of quadrature carriers and $g(t)$ is the input signal pulse.

The M-QAM modulated symbols are passed through the STBC encoder and complex matrix $\mathrm{Z}$ is generated such that symbols are coded through space and time. So, replicas of modulated symbols for Alamouti coding are sent through two transmit antennas and over two time slots.

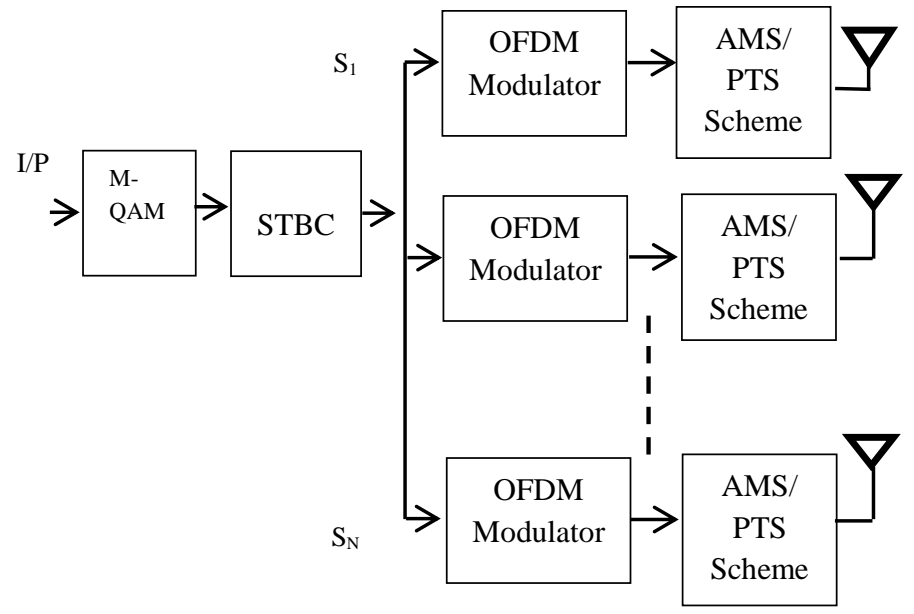

Fig. 1: Block diagram of STBC MIMO OFDM system with AMS/PTS Scheme.

The encoded sequence can be found by

$$
Z=\left[\begin{array}{cc}
z_{0} & -z_{1} * \\
z_{1} & z_{0} *
\end{array}\right\rfloor
$$

The encoded bits are given to the OFDM modulator where the bits are mapped with the orthogonal carriers. An inverse FFT is computed on each set of symbols, giving a set of complex time-domain samples.

$$
z(n)=\frac{1}{\sqrt{N}} \sum_{k=0}^{N-1} Z(k) e^{\frac{j 2 \pi n k}{N}}
$$

where $\mathrm{j}=\sqrt{-1}$ and $\mathrm{n}=0,1, \ldots . .(\mathrm{N}-1)$.

After OFDM modulation, AMS or PTS scheme is applied to reduce PAPR. Finally, the signal with minimum PAPR is transmitted through its respective antennas.

The PAPR of MIMO-OFDM system is defined by

$$
\operatorname{PAPR}(z(n))=\frac{\max \left\{|z(n)|^{2}\right\}}{E\left\{|z(n)|^{2}\right\}}
$$

where $\mathrm{E}\{$.$\} is the mathematical expectation.$

Complementary cumulative density function (CCDF) for PAPR is given by

$$
\operatorname{CCDF}(\operatorname{PAPR}(z(n)))=P_{r}\left(\operatorname{PAPR}(z(n))>P A P R_{0}\right)
$$

\section{METHODS}

\subsection{AMS Scheme}

The AMS scheme is proposed by Tao Jiang and Cai $\mathrm{Li}$ in 2012 [12]. In this scheme, after STBC encoder, the coded data is partitioned into sub blocks, and IFFT operation is performed on each sub block where the frequency domain signals are converted into time domain signals.

Finally, AMS scheme is implemented, in which two inputs are given to the AMS block one input is from IFFT block and another input to AMS block is the conjugate of the output of the IFFT block. Suppose the output of the IFFT block is $y_{1}^{m},(m=0,1 \ldots . M-1)$, then the two inputs to the AMS block will be $t_{1}^{m}$ and $t_{2}^{m}$ where,

$$
t_{2}^{m}=\left(t_{1}^{m}\right)^{*}
$$

The AMS scheme will generate new sequences which are given by

$$
\begin{aligned}
& t_{1}^{\prime}=a^{m}\left(t_{1}^{m}\right)^{c^{m}}+b^{m} t_{2}^{m} \\
& t_{1}^{\prime}=a^{m}\left(t_{2}^{m}\right)^{c^{m}}-b^{m} t_{1}^{m}
\end{aligned}
$$

Where $a^{m}$ and $b^{m}$ are positive integers with $a^{m} \neq 0$ and $c^{m}$ $=1$ and 2 respectively.

Then the alternate transmitted signals are given by

$$
t_{i}=\sum_{m=0}^{M-1} t_{i}^{m}
$$

where $i=1,2$.

Finally, the signal with the lowest PAPR are chosen for transmission.

\subsection{PTS Scheme}

The partial transmit sequence (PTS) is an attractive technique because of good PAPR reduction performance and no restriction to the number of subcarriers [13]. It can be said that PTS method is a modified method of SLM. PTS method works better than SLM method. The main advantage of this scheme is that there is no need to send any side information to the receiver of the system, when, differential modulation is applied in all sub blocks. In this scheme, the coming input bits are divided into smaller disjoint sub blocks. Input from each partitioned sub block converted from frequency domain to time domain by using $\mathrm{N}$-point inverse fast fourier transform (IFFT). The time domain sequences are multiplied by rotating phase factors, $z=\left[z_{1} z_{2} \ldots z_{m}\right]^{T}$, to minimize PAPR and then these sequences are then added to form the OFDM symbol for transmission.

The resulting time domain signal,

Allowable phase factor,

$$
x^{\prime}(z)=\sum_{m=1}^{M} z_{m} \cdot x_{m}
$$

$$
z_{m}=e^{j \Phi_{m}}
$$

$x_{m}$ is the time domain sequence and $\Phi_{m}$ can take the value between $(0,2 \pi)$.

The main aim of this scheme is to design an optimal phase factor for each sub block set that minimizes the PAPR. Finally, the signal with the lowest PAPR are chosen for transmission.

\section{SIMULATION AND RESULTS}

Simulations have been conducted to evaluate the PAPR reduction in STBC MIMO OFDM system where number of subcarriers, $\mathrm{N}=256$, oversampling factor, $\mathrm{Nos}=4$, number of subblocks, $\mathrm{M}=2 \& 4$, and number of OFDM blocks for iteration, Nblk $=3000$. Complementary cumulative density function is used to depict the statistical properties of PAPR in STBC MIMO OFDM system, as PAPR is a random variable. 


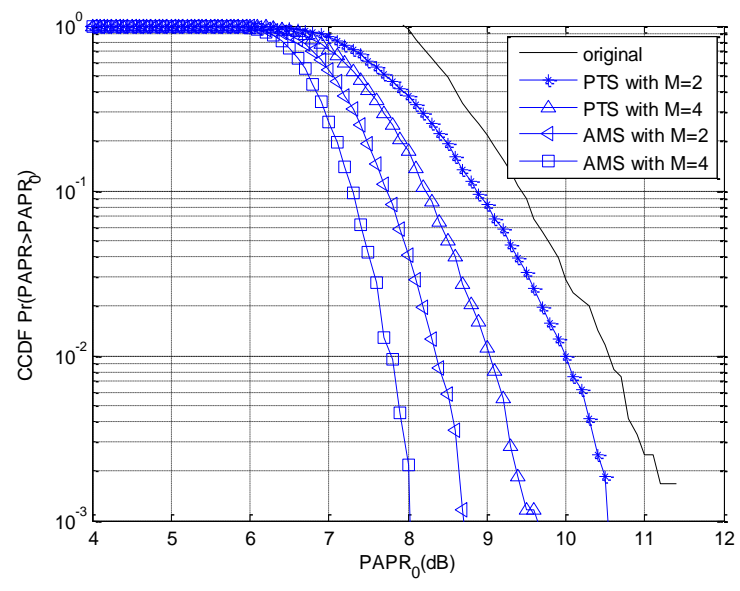

Fig.2: PAPR reduction of AMS and PTS scheme with 4-QAM.

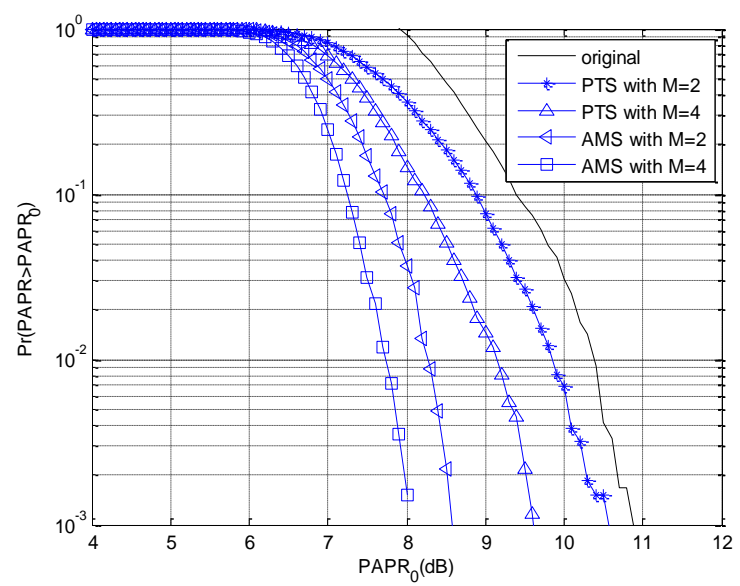

Fig.3: PAPR reduction of AMS and PTS scheme with 8-QAM.

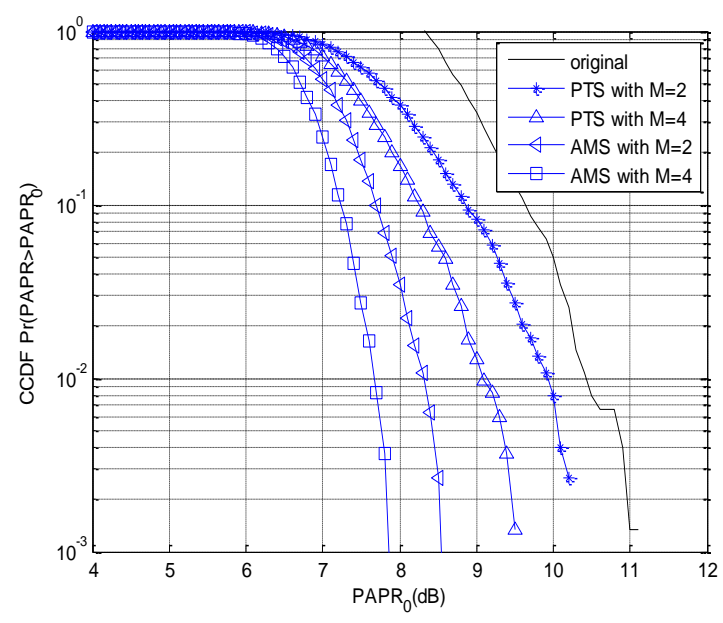

Fig.4: PAPR reduction of AMS and PTS scheme with 16-QAM.

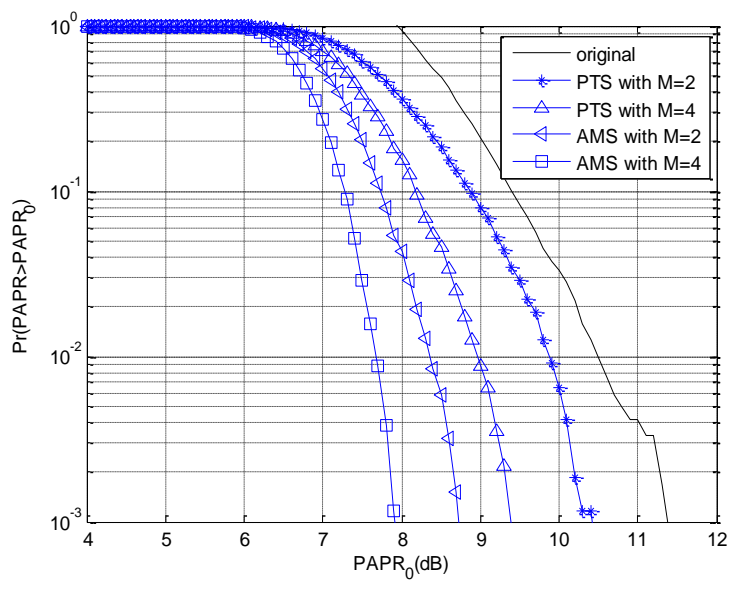

Fig.5: PAPR reduction of AMS and PTS scheme with 32-QAM.

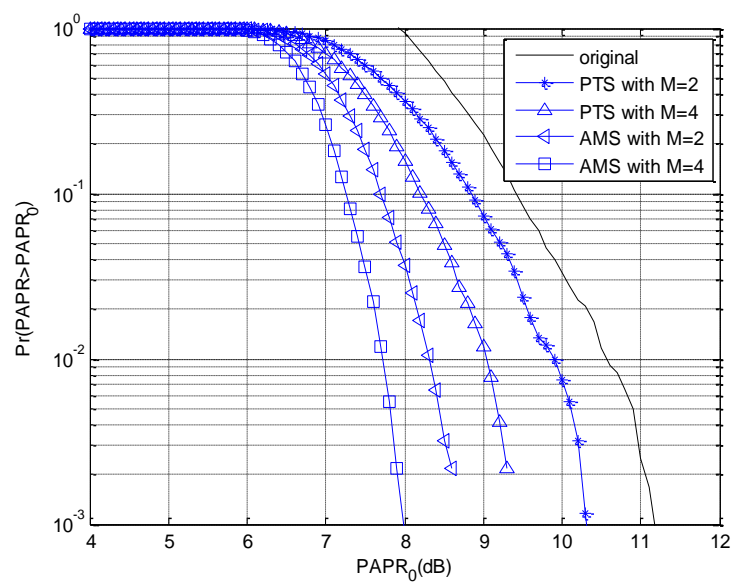

Fig.6: PAPR reduction of AMS and PTS scheme with 64-QAM.

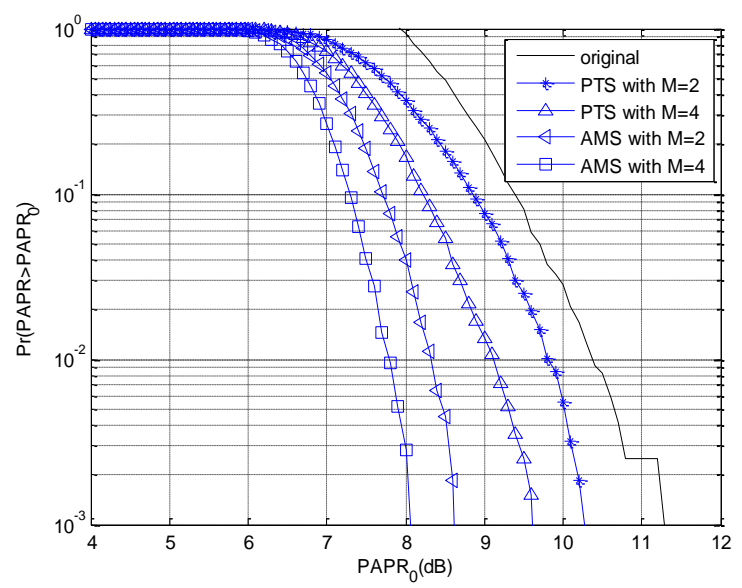

Fig.7: PAPR reduction of AMS and PTS scheme with 128-QAM.

Figure 2, figure 3, figure 4, figure 5, figure 6 and figure 7 show the reduction of PAPR with AMS/PTS with $M=2$ and 4, and original signal for 4-QAM, 8-QAM, 16-QAM, 32-QAM, 64-QAM and 128-QAM. In this PTS scheme is applied with the number of phase rotation factors for each subblock, i.e. W is equal to the number of subblocks, which means for $M=2$, $\mathrm{W}=2$ and for $\mathrm{M}=4, \mathrm{~W}=4$, respectively. Table $\mathrm{I}$ shows the different values of PAPR at $\mathrm{P}_{\mathrm{r}}\left(\mathrm{PAPR}>\mathrm{PAPR}_{0}\right)=10^{-2}$. For 4$\mathrm{QAM}$, when the original signal is compared with the AMS 
scheme, $1.5097 \mathrm{~dB}$ and $2.7621 \mathrm{~dB}$ PAPR reduction with $\mathrm{M}=2$ and $\mathrm{M}=4$, whereas when the original signal is compared with the PTS scheme, $0.545 \mathrm{~dB}$ and $2.1871 \mathrm{~dB}$ PAPR reduction with $\mathrm{M}=2$ and $\mathrm{M}=4$. For $8-\mathrm{QAM}$, when the original signal is compared with the AMS scheme, $1.2346 \mathrm{~dB}$ and $2.6464 \mathrm{~dB}$ PAPR reduction with $M=2$ and $M=4$, whereas when the original signal is compared with the PTS scheme, $0.53 \mathrm{~dB}$ and $2.1093 \mathrm{~dB}$ PAPR reduction with $\mathrm{M}=2$ and $\mathrm{M}=4$.

Table 1. Comparison of AMS and PTS schemes with original signal for different modulation schemes

\begin{tabular}{|c|c|c|c|c|c|}
\hline $\mathbf{M}$ & Modulation & CCDF & $\begin{array}{c}\text { Original } \\
\text { (dB) }\end{array}$ & $\begin{array}{l}\text { PTS } \\
\text { (dB) }\end{array}$ & $\begin{array}{c}\text { AMS } \\
\text { (dB) }\end{array}$ \\
\hline \multirow{6}{*}{2} & 4-QAM & $10^{-2}$ & 10.545 & 10 & 9.0353 \\
\hline & 8-QAM & $10^{-2}$ & 10.38 & 9.85 & 9.1454 \\
\hline & 16-QAM & $10^{-2}$ & 10.4224 & 9.9224 & 9.0885 \\
\hline & 32-QAM & $10^{-2}$ & 10.5 & 9.872 & 8.9643 \\
\hline & 64-QAM & $10^{-2}$ & 10.564 & 9.892 & 9.0408 \\
\hline & 128-QAM & $10^{-2}$ & 10.374 & 9.805 & 9.12 \\
\hline \multirow{6}{*}{4} & 4-QAM & $10^{-2}$ & 10.545 & 8.3579 & 7.7829 \\
\hline & 8-QAM & $10^{-2}$ & 10.38 & 8.2707 & 7.7336 \\
\hline & 16-QAM & $10^{-2}$ & 10.4224 & 8.3124 & 7.6729 \\
\hline & 32-QAM & $10^{-2}$ & 10.5 & 8.3605 & 7.6788 \\
\hline & 64-QAM & $10^{-2}$ & 10.564 & 8.31 & 7.722 \\
\hline & 128-QAM & $10^{-2}$ & 10.374 & 8.32 & 7.7879 \\
\hline
\end{tabular}

For 16-QAM, when the original signal is compared with the AMS scheme, $1.3339 \mathrm{~dB}$ and $2.7495 \mathrm{~dB}$ PAPR reduction with $M=2$ and $M=4$, whereas when the original signal is compared with the PTS scheme, $0.5 \mathrm{~dB}$ and $2.11 \mathrm{~dB}$ PAPR reduction with $M=2$ and $M=4$. For 32-QAM, when the original signal is compared with the AMS scheme, $1.5357 \mathrm{~dB}$ and $2.8212 \mathrm{~dB}$ PAPR reduction with $M=2$ and $M=4$, whereas when the original signal is compared with the PTS scheme, $0.628 \mathrm{~dB}$ and $2.1395 \mathrm{~dB}$ PAPR reduction with $\mathrm{M}=2$ and $\mathrm{M}=4$. For 64QAM, when the original signal is compared with the AMS scheme, $1.5232 \mathrm{~dB}$ and $2.842 \mathrm{~dB}$ PAPR reduction with $\mathrm{M}=2$ and $M=4$, whereas when the original signal is compared with the PTS scheme, $0.672 \mathrm{~dB}$ and $2.254 \mathrm{~dB}$ PAPR reduction with $\mathrm{M}=2$ and $\mathrm{M}=4$. For $128-\mathrm{QAM}$, when the original signal is compared with the AMS scheme, $1.254 \mathrm{~dB}$ and $2.5861 \mathrm{~dB}$ PAPR reduction with $M=2$ and $M=4$, whereas when the original signal is compared with the PTS scheme, $0.569 \mathrm{~dB}$ and 2.054 $\mathrm{dB}$ PAPR reduction with $\mathrm{M}=2$ and $\mathrm{M}=4$.

\section{CONCLUSION}

Orthogonal frequency division multiplexing (OFDM) is a very attractive technique for wireless communications due to its spectrum efficiency and channel robustness. One of the major drawbacks of in MIMO-OFDM systems is that the transmitted signal exhibits a high PAPR when the input sequences are correlated. In the present work, two different PAPR reduction techniques i.e. AMS and PTS have been implemented on the MIMO OFDM system and the PAPR reduction parameter has been analyzed. The Alamouti's STBC has been used to make MIMO OFDM system. The results show that AMS scheme is more effective than the PTS scheme to reduce PAPR in STBC MIMO OFDM systems with QAM modulation.

\section{FUTURE SCOPE}

Further this work can be extended to increase the performance of the MIMO-OFDM system by using different channel coding techniques and using other PAPR reduction techniques. Also there is a scope to implement the MIMOOFDM system by using different modulation schemes.

\section{REFERENCES}

[1] Zhang W. and. Letaief, K. B, "Space-time/frequency coding for MIMO-OFDM in next generation broadband wireless systems", IEEE Wireless Communications, vol. 14, (2007) 32-43.

[2] Bolcskei, H. and Zurich, E., "MIMO-OFDM wireless systems: basics, perspectives, and challenges" IEEE Wireless Communications, (Aug. 2006) 31-37.

[3] Paulraj, A. J., Nabar, R. U. and Gore, D. A., 2003 , "Introduction to Space-Time Wireless Communications" Cambridge, UK: Cambridge Univ. Press.

[4] Agrawal, D., Tarokh, V., Naguib, A. and Seshadri, N., (1998), "Space-time coded OFDM for high data-rate wireless communication over wideband channels", IEEE Conference on Vehicular Technology, vol.3, (1998) 2232-2236.

[5] Uysal, M., Dhahir, N. A. and Georghiades, C. N., "A space-time block-coded OFDM scheme for unknown frequency-selective fading channels," IEEE Communications Letters, vol. 5, (2001) 393-395.

[6] Saglam, H. D., 2004, "Simulation Performance of Multiple-Input Multiple-Output Systems Employing Single-Carrier Modulation and Orthogonal Frequency Division Multiplexing", Master's Thesis, Naval Postgraduate School, Monterey, California.

[7] Zhang, Q., Yu, F. and Nallanathan, A. "Improving achievable rates in MPSK amplify-and-forward relay networks via clipping," IEEE Trans. Veh. Technol., vol. 59, no. 5, (Jun. 2010) 2133-2137.

[8] Naeiny, M. F. and Marvasti, F. "Selected mapping algorithm for PAPR reduction of space-frequency coded OFDM systems without side information," IEEE Trans. Veh. Technol., vol. 60, no. 3, (Mar. 2011) $1211-1216$

[9] Du, Z., Beaulieu, N. C. and Zhu, J. "Selective timedomain filtering for reduced-complexity PAPR reduction in OFDM," IEEE Trans. Veh. Technol., vol. 58, no. 3, (Mar. 2009) 1170-1176.

[10] Ghassemi, A. and Gulliver, T. A. "A low-complexity PTS-based radix FFT method for PAPR reduction in OFDM systems," IEEE Trans. Signal Process, vol. 56, no. 3, ( Mar. 2008) 1161-1166.

[11] Rodrigues, M. R. D. and Wassell, I. J. "IMD reduction with SLM and PTS to improve the error-probability performance of nonlinearly distorted OFDM signals," IEEE Trans. Veh. Technol., vol. 55, no. 2, (Mar. 2006) 537-548.

[12] Jiang, T. and Li, C. "Simple Alternative Multisequences for PAPR Reduction Without Side Information in SFBC MIMO-OFDM Systems" IEEE Trans. Veh. Technol., vol. 61, no. 7, (Sept. 2012), 3311-3315. 